\title{
Prescription pattern of antiepileptic drugs in a tertiary care hospital
}

\author{
Anu Mohan T., Anuradha M.*
}

Department of Pharmacology, Government Medical College, Kozhikode, Kerala, India

Received: 30 January 2020

Accepted: 07 March 2020

*Correspondence:

Dr. Anuradha M.,

Email: dranuradha.m1@gmail.com

Copyright: (C) the author(s), publisher and licensee Medip Academy. This is an open-access article distributed under the terms of the Creative Commons Attribution Non-Commercial License, which permits unrestricted non-commercial use, distribution, and reproduction in any medium, provided the original work is properly cited.

\begin{abstract}
Background: Epilepsy is a chronic neurological disorder which affects about $0.5 \%$ to $1 \%$ of the population. The older antiepileptic drugs like carbamazepine and phenytoin are the mainstay of treatment of epilepsy. With the development of newer drugs for various type of epilepsy, the prescribing pattern for epilepsy has changed over the last decade. The objective of this study was to evaluate the prescription pattern of antiepileptic drugs in outpatient department of a tertiary care health care centre in Kerala and to assess how adherent they are to the available treatment guidelines of epilepsy.

Methods: Data was collected from patients attending the outpatient department of Neurology in Government Medical College, Kozhikode for 2 months from January 2018 to February 2018. A total of 442 patients were enrolled in the study and data regarding the type of epilepsy, the antiepileptic drug prescribed, and the demographic profile were recorded and analysed.

Results: Among the study participants, 237 were males (53.6\%) and 205 females (46.2\%). The study showed that among the 442 participants enrolled, the most common type of epilepsy was focal seizures $(64.5 \%)$ and the most commonly prescribed drug was carbamazepine (28\%), followed by levetiracetam (22\%) and valproate (20\%). Majority of the patients were treated with a single drug (79.2\%).

Conclusions: Newer drugs have been increasingly added to the list of antiepileptic drugs, but most of them serve as adjuvant to older ones and the important drugs used as monotherapy are still the older ones.
\end{abstract}

Keywords: Antiepileptic drugs, Epilepsy, Prescription pattern

\section{INTRODUCTION}

According to the International League against epilepsy, epilepsy is characterised by recurrent two or more seizures unprovoked by any immediate identifiable cause. ${ }^{1}$ Epilepsy affects about $0.5 \%$ to $1 \%$ of the population worldwide., ${ }^{2,3}$ Prevalence of active epilepsy worldwide is 4-5 per 1000 population and in India it is 47 per 1000 population. ${ }^{4}$

Prescription pattern monitoring studies are a tool for assessing the prescribing pattern of medicines and they help in promoting appropriate use of drugs and thus reduce misuse of drugs. This study is to assess the standards of medical treatment for epilepsy at tertiary level of health care. ${ }^{5}$ Antiepileptic drugs (AEDs) are the mainstay of treatment of epilepsy and are prescribed to patients of all ages worldwide mostly as monotherapy. The prescribing pattern of Antiepileptic drugs has changed over the last decade with the development of newer drugs which have improved safety, tolerability and patient acceptability. ${ }^{6,7}$ This study has been undertaken to evaluate the prescription pattern of antiepileptic drugs for various type of epilepsies in Govt. Medical College, Kozhikode. In a study conducted by Thasni et al, they concluded that newer AEDs were commonly used as compared to Conventional AEDs and the most commonly used AED was Levetiracetam. ${ }^{8}$ In another study 
conducted by Eswari et al, it was concluded that conventional drugs like Phenytoin is a commonly prescribed drug to treat seizures followed by Sodium valproate. ${ }^{9}$

Objective was to evaluate the prescription pattern of Antiepileptic drugs in a tertiary care hospital, and to evaluate how rational is the prescription for various epilepsies.

\section{METHODS}

It was an observational study and 442 patients attending the outpatient department of Neurology department of Govt. Medical College for a period from January 2018 to February 2018 were enrolled.

\section{Inclusion criteria}

Patients of all age groups diagnosed with epilepsy and patients who had given informed consent were included.

\section{Exclusion criteria}

Patients with uncertain diagnosis and did not give consent were excluded.

Written informed consent was taken from the patient or legally authorised representative before starting the study. Assent was taken in case of children more than 12 and less than 18 years of age.

\section{Procedure}

Subjects who fulfilled the inclusion and exclusion criteria, and those who gave a written informed consent were enrolled in the study. The proforma was filled using the patients prescription data. The data was entered in excel and analysed using SPSS software.

\section{RESULTS}

A total of 442 patients were enrolled in the study.

Table 1: Sex distribution of the study population.

\begin{tabular}{|lll|}
\hline Sex & Number & Percentage $(\%)$ \\
\hline Male & 238 & 53.8 \\
\hline Female & 204 & 46.1 \\
\hline
\end{tabular}

There was a male preponderance in the study.

Table 2: Age wise distribution of the study population.

\begin{tabular}{|ll|}
\hline Age group (years) & Percentage (\%) \\
\hline $\mathbf{0 - 1 2}$ & 39.6 \\
\hline $\mathbf{1 3 - 2 5}$ & 28.6 \\
\hline $\mathbf{2 6 - 5 0}$ & 23.3 \\
\hline $\mathbf{5 0}$ & 8.5 \\
\hline
\end{tabular}

The analysis of age groups of the patients showed that the most common age group was $0-12$ years $(39.6 \%)$ and the least common age group was above 50 years of age $(8.5 \%)$.

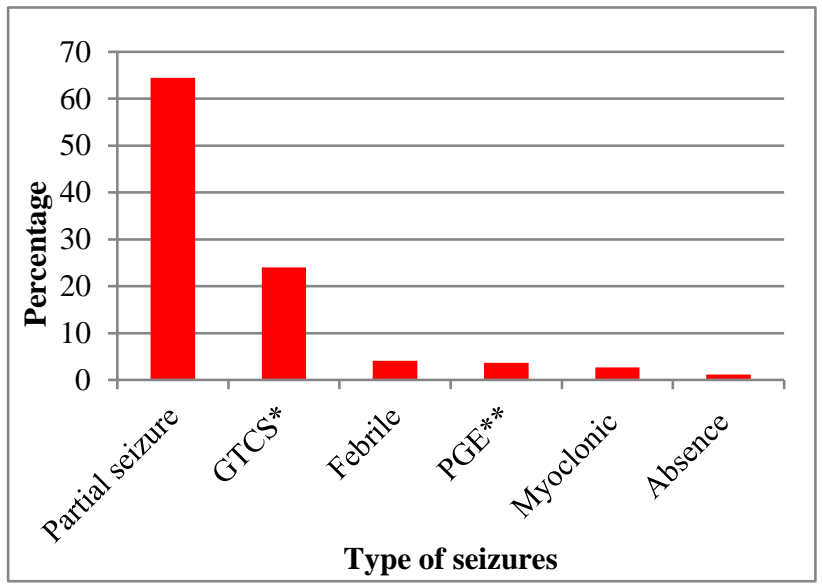

Figure 1: Type of seizure distribution in the study population.

*GTCS- Generalised tonic clonic seizures; **PGE- Primary generalised epilepsy

The analysis of the type of seizure showed that the most common type was partial seizures $(64.5 \%)$ and the least common type was absence seizures $(1.19 \%)$.

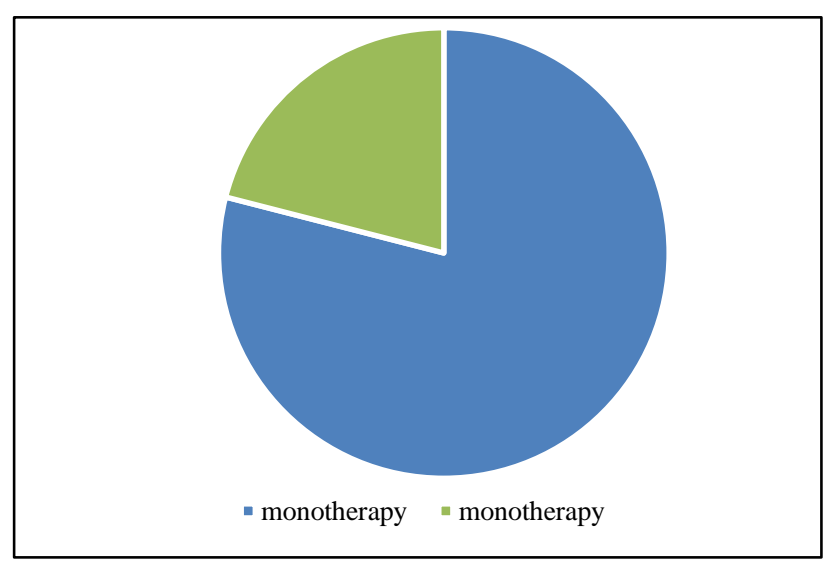

Figure 2: Mode of therapy.

Regarding the mode of therapy, majority of the patients were treated with monotherapy $(79 \%)$. Polytherapy was given for $21 \%$ of the study population. Monotherapy was with conventional drugs carbamazepine, valproate and levetiracetam and polytherapy was given along with conventional drugs with newer drugs like levetiracetam, oxcarbazepine, lamotrigine and topiramate.

The analysis of prescriptions showed that the most commonly prescribed drug was carbamazepine (35\%) followed by valproate $(25 \%)$ and levetiracetam $(22 \%)$. The other drugs prescribed were phenytoin (6\%), phenobarbitone $(5 \%)$, benzodiazepines $(5 \%)$. The least 
commonly prescribed drugs were the newer drugs like topiramate and lamotrigine $(2 \%)$.

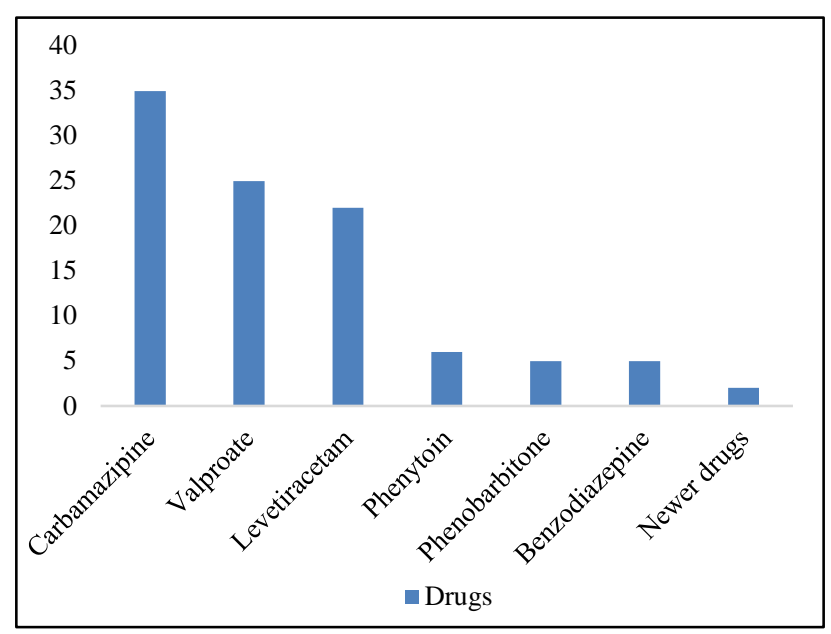

Figure 3: Percentage of individual drugs prescribed.

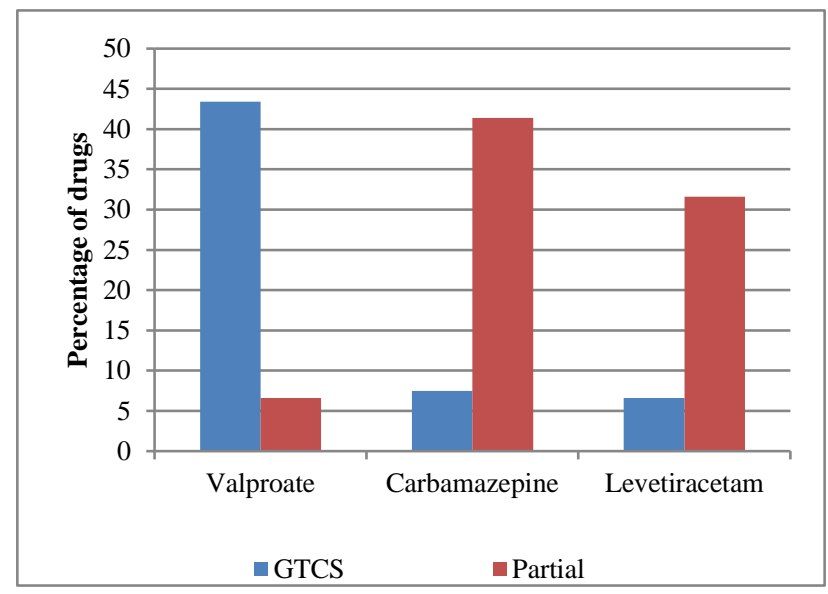

Figure 4: Common drugs prescribed for GTCS and partial seizures.

The most commonly prescribed drug for GTCS was valproate $(43.4 \%)$, followed by carbamazepine $(7.5 \%)$ and levetiracetam (6.6\%). In partial seizures, carbamazepine was the most common drug prescribed $(41.4 \%)$, followed by levetiracetam (31.6\%) and Valproate $(6.6 \%)$.

\section{DISCUSSION}

There are many drugs available in the market for management of epilepsy and many are in the pipeline. Many drugs have come to the market and were later withdrawn due to serious toxicities like Felbamate. $80 \%$ of epilepsies are controlled by treatment but $20 \%$ are still refractory to treatment. In refractory cases, polytherapy is employed in an attempt to reduce the number and severity of seizures. Optimal polytherapy involves combining drugs that differ in mechanism of action and toxicity. ${ }^{10}$ Marginal reduction in the number of seizures will increase quality of life of these patients with epilepsy.
Antiepileptic drugs are notorious for drug-drug interactions because of CYP 450 enzyme induction and inhibition. Potent CYP450 enzymatic inducers include carbamazepine, phenytoin, and Phenobarbital whereas valproic acid is a potent enzyme inhibitor. Interactions with these drugs can occur with concurrent medications that are metabolised by hepatic microsomal enzymes. ${ }^{11}$ Thus, a rational prescribing with AED is very important for effective and safe management of epilepsy and thus has a huge impact on the quality of life of these patients.

While assessing the demographic profile, the most common age group affected was 0- 12 years (39.6\%) and the least affected age group was above 50 years of age $(8.5 \%)$ contrary to the finding in study by Hurtz et al, which said epilepsy was more prevalent in children and in age group above $55 .^{12}$ The analysis on the type of seizure revealed that the most common type was partial seizures $(64.5 \%)$ and the least common type was absence seizures $(1.19 \%)$, but in a study by Gupta et al, majority of patients were affected with generalised tonic-clonic seizure $(71.71 \%)$, followed by idiopathic $(70.03 \%)$ and complex partial seizure $(12.79 \%) .{ }^{13}$

In this study, 79\% were treated with monotherapy and the analysis of prescriptions showed that the most commonly prescribed drug was carbamazepine $(35 \%)$ followed by valproate $(25 \%)$ and levetiracetam $(22 \%)$. the other drugs prescribed were phenytoin $(6 \%)$, phenobarbitone $(5 \%)$ and the least commonly prescribed drugs were topiramate $(1 \%)$ and lamotrigine (1\%).

According to the present study, the most common drug prescribed for GTCS was Valproate and that for partial seizures was carbamazepine. Meta-analysis of different studies and NICE guidelines showed that carbamazepine and lamotrigine are the most suitable first line options for individuals with partial onset seizures and levetiracetam can also be considered for the same. Results also support the use of sodium valproate as the first-line drug for individuals with generalised tonic-clonic seizures and lamotrigine and levetiracetam were suitable alternatives. ${ }^{14}$

Indian guidelines on epilepsy suggests carbamazepine, oxcarbamazepine, phenytoin, valproate and phenobarbitone as first line agents for partial seizures and valproate, phenytoin, phenobarbitone and carbamazepine as first line drug for generalized tonic clonic seizures. ${ }^{15}$

Comparing the study data with international and national guidelines we conclude that the prescription pattern for Antiepileptic drugs is in accordance with the standard guidelines.

\section{CONCLUSION}

Epilepsy is a condition which needs prolonged treatment with antiepileptics and hence the appropriateness of therapy has a great impact on the quality of life of patients. Older antiepileptic agents like carbamazepine, 
valproate, phenytoin are still the most commonly agents as monotherapy whereas newer ones like levetiracetam are mostly used as add on drug in cases of treatment failure with older drugs. Antiepileptic prescribing in this study population is in accordance to the standard treatment guidelines for epilepsy. Further studies regarding the safety of these drugs in the study population can be done in future to ascertain these results.

Funding: No funding sources

Conflict of interest: None declared

Ethical approval: The study was approved by the Institutional Ethics Committee

\section{REFERENCES}

1. Hauser WA, Kurland LT. The epidemiology of epilepsy in Rochester, Minnesota, 1935 through 1967. Epilepsia. 1975;16:1-66.

2. De Boer HM, Mula M, Sander JW. The global burden and stigma of epilepsy. Epilepsy Behav. 2008;12(4):540-6.

3. Council JE. Epilepsy prevalence, incidence and other statistics. UK and Ireland: Joint Epilepsy Council. 2011.

4. Murthy VN, Anusha B, Perumal P. A study on trends in prescribing pattern of anti-epileptic drugs in teritiary care teaching hospital. Int $\mathbf{J}$ Chem Pharm Sci. 2012;3(2):25-32.

5. Sridharan R. Special section: recent advances in epilepsy epidemiology of epilepsy. Curr Sci. 2002;82;664-70.

6. Groot MC, Schuerch M, Vries F, Hesse U, Oliva B, Gil M, et al. Antiepileptics drug use in seven electronic health record databases in Europe: a methodologic comparison. Epilepsia. 2014;55(5):666-73.

7. Stephen LJ, Brodie MJ. Antiepileptic drug monotherapy versus polytherapy: pursuing seizure freedom and tolerability in adults. Curr Opin Neurol. 2012;25(2):164-72.

8. Thasni K, Abdurahiman P, Sadan SE. Assessment of prescription pattern and quality of life in patient with epilepsy: a tertiary care hospital based prospective study. Int J Med Health Res. 2017;3(10):106-11.

9. Eswari PVSN, Kumar PB, Lakshmi P. An observational study on prescribing pattern of antiepileptic drugs in pediatric patients at a tertiary care hospital. World J Pharma Med Res. 2017;3(7):223-6.

10. Perucca E, Gram L, Avanzini G, Dulac O. Antiepileptic drugs as a cause of worsening seizures. Epilepsia. 1998;39(1):5-17.

11. Smith TR, Wagner M, Liu MT. Effective drug therapy for epilepsy. Pharmacy Times. 2012:13(1):23.

12. Hirtz D, Thurman DJ, Gwinn-Hardy K, Mohamed M, Chaudhuri AR, Zalutsky R. How common are the "common" neurologic disorders?. Neurology. 2007;68(5):326-37.

13. Gupta A, Desai C. Profile of epilepsy in a tertiary care public sector hospital of western India. Int $\mathrm{J}$ Community Med Public Health. 2017;4(7):67-8.

14. Nevitt SJ, Sudell M, Weston J, Smith CT, Marson AG. Antiepileptic drug monotherapy for epilepsy: a network meta-analysis of individual participant data. Cochrane Database Syst Rev. 2017(6).

15. Roy MK, Das D. Indian guidelines on epilepsy. IAP expert committee guidelines. 2013: 528-532.

Cite this article as: Anu MT, Anuradha M. Prescription pattern of antiepileptic drugs in a tertiary care hospital. Int J Basic Clin Pharmacol 2020;9:63841. 